ACCEPTED By The Astrophysical Journal

Preprint typeset using LTEX style emulateapj v. 08/22/09

\title{
Fermi-LAT OBSERVATIONS OF TWO GAMMA-RAY EMISSION COMPONENTS FROM THE QUIESCENT SUN
}

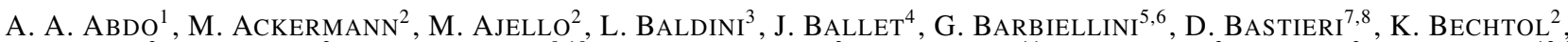
R. Bellazzini ${ }^{3}$, B. BerenJi ${ }^{2}$, E. Bonamente ${ }^{9,10}$, A. W. Borgland ${ }^{2}$, A. Bouvier ${ }^{11}$, J. BREgEON $^{3}$, A. BREZ $^{3}$, M. BRIGIDA ${ }^{12,13,14}$, P. BRuel ${ }^{15}$, R. Buehler ${ }^{2}$, S. Buson ${ }^{7,8}$, G. A. CAliANDRo ${ }^{16}$, R. A. CAMERON ${ }^{2}$, P. A. CARAVEO ${ }^{17}$, J. M. CASANDJiAN ${ }^{4}$ C. CeCChi ${ }^{9,10}$, E. Charles ${ }^{2}$, A. CheKhtman ${ }^{18}$, J. ChiAng ${ }^{2}$, S. Ciprini ${ }^{10}$, R. Claus ${ }^{2}$, J. Cohen-Tanugi ${ }^{19}$, J. Conrad ${ }^{20,21,22}$ S. Cutini ${ }^{23}$, A. De Angelis ${ }^{24}$, F. De PAlma ${ }^{12,13}$, C. D. Dermer ${ }^{25}$, S. W. Digel ${ }^{2}$, E. Do Couto E Sillva ${ }^{2}$, P. S. DRell ${ }^{2}$,

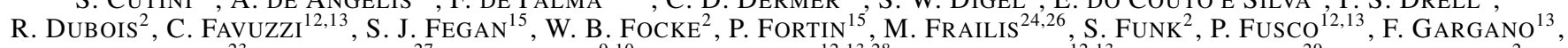

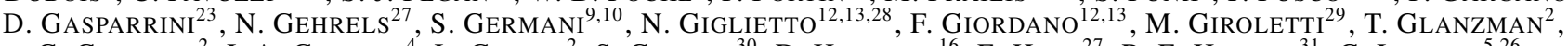

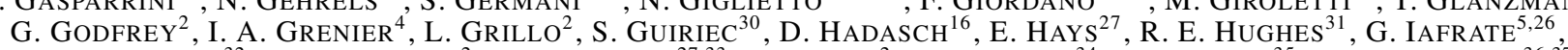
G. Jóhannesson ${ }^{32}$, A. S. Johnson ${ }^{2}$, T. J. JOHNSON ${ }^{27,33}$, T. KAMAE ${ }^{2}$, H. KATAGIRI ${ }^{34}$, J. KATAOKA ${ }^{35}$, J. KNÖDLSEDER ${ }^{36,37}$,

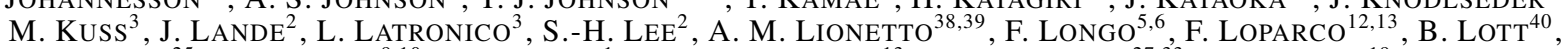

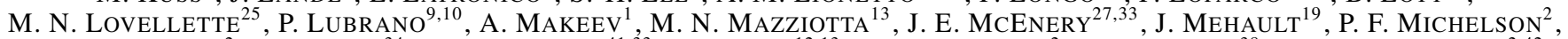
W. Mitthumsiri ${ }^{2}$, T. Mizuno ${ }^{34}$, A. A. Moiseev ${ }^{41,33}$, C. Monte ${ }^{12,13}$, M. E. Monzani ${ }^{2}$, A. Morselli ${ }^{38}$, I. V. Moskalenko ${ }^{2,42}$,

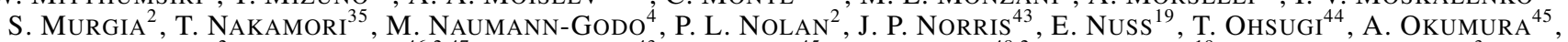
N. OMOdeI ${ }^{2}$, E. ORlando ${ }^{46,2,47}$, J. F. ORmes ${ }^{43}$, M. OZAKi ${ }^{45}$, D. PAneque ${ }^{48,2}$, V. Pelassa ${ }^{19}$, M. Pesce-Rollins ${ }^{3}$, M. Pierbattista ${ }^{4}$, F. PIRON ${ }^{19}$, T. A. PORTER ${ }^{2}$, S. RAInò ${ }^{12,13}$, R. RANDO ${ }^{7,8}$, M. RAZZANO $^{3}$, A. ReIMER $^{49,2}$, O. REIMER $^{49,2}$, T. Reposeur ${ }^{40}$, S. Ritz ${ }^{11}$, H. F.-W. SAdrozinski ${ }^{11}$, T. L. SChALK ${ }^{11}$, C. SGrò ${ }^{3}$, G. H. Share ${ }^{50}$, E. J. Siskind ${ }^{51}$, P. D. SMITH ${ }^{31}$,

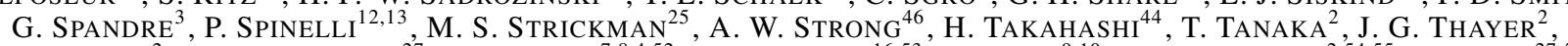

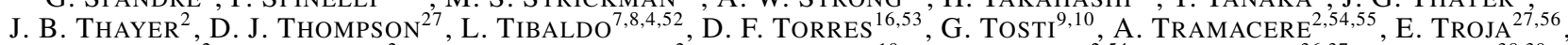

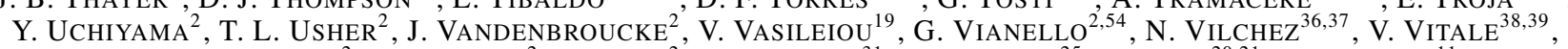

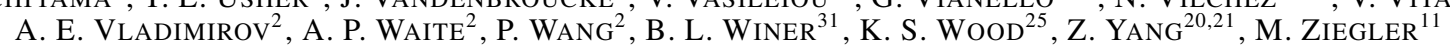

Accepted by The Astrophysical Journal

\section{ABSTRACT}

We report the detection of high-energy $\gamma$-rays from the quiescent Sun with the Large Area Telescope (LAT) on board the Fermi Gamma-Ray Space Telescope (Fermi) during the first 18 months of the mission. These observations correspond to the recent period of low solar activity when the emission induced by cosmic rays is brightest. For the first time, the high statistical significance of the observations allows clear separation of the two components: the point-like emission from the solar disk due to cosmic ray cascades in the solar atmosphere, and extended emission from the inverse Compton scattering of cosmic ray electrons on solar photons in the heliosphere. The observed integral flux $(\geq 100 \mathrm{MeV})$ from the solar disk is $(4.6 \pm 0.2 \text { [statistical error }]_{-0.8}^{+1.0}$ [systematic error] $) \times 10^{-7} \mathrm{~cm}^{-2} \mathrm{~s}^{-1}$, which is $\sim 7$ times higher than predicted by the "nominal" model of Seckel et al. (1991). In contrast, the observed integral flux $(\geq 100 \mathrm{MeV})$ of the extended emission from a region of $20^{\circ}$ radius centered on the Sun, but excluding the disk itself, $\left(6.8 \pm 0.7\right.$ [stat.] ${ }_{-0.4}^{+0.5}$ [syst.] $) \times 10^{-7} \mathrm{~cm}^{-2} \mathrm{~s}^{-1}$, along with the observed spectrum and the angular profile, are in good agreement with the theoretical predictions for the inverse Compton emission.

Subject headings: astroparticle physics — Sun: atmosphere — Sun: heliosphere — Sun: X-rays, gamma rays — cosmic rays - gamma rays: general

\footnotetext{
${ }^{1}$ Center for Earth Observing and Space Research, College of Science, George Mason University, Fairfax, VA 22030, resident at Naval Research Laboratory, Washington, DC 20375

2 W. W. Hansen Experimental Physics Laboratory, Kavli Institute for Particle Astrophysics and Cosmology, Department of Physics and SLAC National Accelerator Laboratory, Stanford University, Stanford, CA 94305, USA

${ }^{3}$ Istituto Nazionale di Fisica Nucleare, Sezione di Pisa, I-56127 Pisa, Italy

${ }^{4}$ Laboratoire AIM, CEA-IRFU/CNRS/Université Paris Diderot, Service d'Astrophysique, CEA Saclay, 91191 Gif sur Yvette, France

${ }^{5}$ Istituto Nazionale di Fisica Nucleare, Sezione di Trieste, I-34127 Trieste, Italy

${ }^{6}$ Dipartimento di Fisica, Università di Trieste, I-34127 Trieste, Italy

${ }^{7}$ Istituto Nazionale di Fisica Nucleare, Sezione di Padova, I-35131 Padova, Italy

${ }^{8}$ Dipartimento di Fisica "G. Galilei", Università di Padova, I-35131 Padova, Italy

${ }^{9}$ Istituto Nazionale di Fisica Nucleare, Sezione di Perugia, I-06123 Perugia, Italy

${ }^{10}$ Dipartimento di Fisica, Università degli Studi di Perugia, I-06123 Perugia, Italy

${ }^{11}$ Santa Cruz Institute for Particle Physics, Department of Physics and Department of Astronomy and Astrophysics, University of California at Santa Cruz, Santa Cruz, CA 95064, USA
}

12 Dipartimento di Fisica "M. Merlin” dell'Università e del Politecnico di Bari, I-70126 Bari, Italy

${ }^{13}$ Istituto Nazionale di Fisica Nucleare, Sezione di Bari, 70126 Bari, Italy

14 email: brigida@ba.infn.it

${ }^{15}$ Laboratoire Leprince-Ringuet, École polytechnique, CNRS/IN2P3, Palaiseau, France

${ }^{16}$ Institut de Ciencies de l'Espai (IEEC-CSIC), Campus UAB, 08193 Barcelona, Spain

${ }^{17}$ INAF-Istituto di Astrofisica Spaziale e Fisica Cosmica, I-20133 Milano, Italy

18 Artep Inc., 2922 Excelsior Springs Court, Ellicott City, MD 21042, resident at Naval Research Laboratory, Washington, DC 20375

${ }^{19}$ Laboratoire Univers et Particules de Montpellier, Université Montpellier 2, CNRS/IN2P3, Montpellier, France

${ }^{20}$ Department of Physics, Stockholm University, AlbaNova, SE-106 91 Stockholm, Sweden

${ }^{21}$ The Oskar Klein Centre for Cosmoparticle Physics, AlbaNova, SE-106 91 Stockholm, Sweden

${ }^{22}$ Royal Swedish Academy of Sciences Research Fellow, funded by a grant from the K. A. Wallenberg Foundation

${ }^{23}$ Agenzia Spaziale Italiana (ASI) Science Data Center, I-00044 Frascati (Roma), Italy

${ }^{24}$ Dipartimento di Fisica, Università di Udine and Istituto Nazionale di 


\section{INTRODUCTION}

The Sun is a well-known source of X-rays and $\gamma$-rays during solar flares (Peterson \& Winckler 1959; Chupp et al. 1973; Kanbach et al. 1993), which are high-energy phenomena associated with the flare-accelerated particle interactions in the solar atmosphere. Quiescent solar $\gamma$-ray emission from hadronic cosmic-ray (CR) interactions with the solar atmosphere and photosphere was first mentioned by Dolan \& Fazio (1965). Peterson et al. (1966) estimated its flux based on measurements of terrestrial emission, and Hudson (1989) suggested

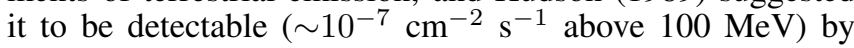
the EGRET experiment on board the Compton Gamma-Ray Observatory (CGRO). The first, and so far the only, detailed theoretical study of $\gamma$-ray emission from interactions of CR protons in the solar atmosphere was published by Seckel et al. (1991). The integral flux above $100 \mathrm{MeV}$ was predicted to be

Fisica Nucleare, Sezione di Trieste, Gruppo Collegato di Udine, I-33100 Udine, Italy

25 Space Science Division, Naval Research Laboratory, Washington, DC 20375, USA

${ }^{26}$ Osservatorio Astronomico di Trieste, Istituto Nazionale di Astrofisica, I-34143 Trieste, Italy

${ }^{27}$ NASA Goddard Space Flight Center, Greenbelt, MD 20771, USA

28 email: nico.giglietto@ba.infn.it

${ }^{29}$ INAF Istituto di Radioastronomia, 40129 Bologna, Italy

${ }^{30}$ Center for Space Plasma and Aeronomic Research (CSPAR), University of Alabama in Huntsville, Huntsville, AL 35899

${ }^{31}$ Department of Physics, Center for Cosmology and Astro-Particle Physics, The Ohio State University, Columbus, OH 43210, USA

32 Science Institute, University of Iceland, IS-107 Reykjavik, Iceland

${ }^{33}$ Department of Physics and Department of Astronomy, University of Maryland, College Park, MD 20742

${ }^{34}$ Department of Physical Sciences, Hiroshima University, HigashiHiroshima, Hiroshima 739-8526, Japan

${ }^{35}$ Research Institute for Science and Engineering, Waseda University, 34-1, Okubo, Shinjuku, Tokyo 169-8555, Japan

${ }^{36}$ CNRS, IRAP, F-31028 Toulouse cedex 4, France

37 Université de Toulouse, UPS-OMP, IRAP, Toulouse, France

${ }^{38}$ Istituto Nazionale di Fisica Nucleare, Sezione di Roma "Tor Vergata", I-00133 Roma, Italy

${ }^{39}$ Dipartimento di Fisica, Università di Roma "Tor Vergata", I-00133 Roma, Italy

${ }^{40}$ Université Bordeaux 1, CNRS/IN2p3, Centre d'Études Nucléaires de Bordeaux Gradignan, 33175 Gradignan, France

${ }^{41}$ Center for Research and Exploration in Space Science and Technology (CRESST) and NASA Goddard Space Flight Center, Greenbelt, MD 20771

42 email: imos@stanford.edu

43 Department of Physics and Astronomy, University of Denver, Denver, CO 80208, USA

${ }^{44}$ Hiroshima Astrophysical Science Center, Hiroshima University, Higashi-Hiroshima, Hiroshima 739-8526, Japan

45 Institute of Space and Astronautical Science, JAXA, 3-1-1 Yoshinodai, Chuo-ku, Sagamihara, Kanagawa 252-5210, Japan

${ }^{46}$ Max-Planck Institut für extraterrestrische Physik, 85748 Garching, Germany

47 email: eorlando@stanford.edu

48 Max-Planck-Institut für Physik, D-80805 München, Germany

${ }^{49}$ Institut für Astro- und Teilchenphysik and Institut für Theoretische Physik, Leopold-Franzens-Universität Innsbruck, A-6020 Innsbruck, Austria

${ }^{50}$ Department of Astronomy, University of Maryland, College Park, MD 20742, resident at Naval Research Laboratory, Washington, DC 20375

51 NYCB Real-Time Computing Inc., Lattingtown, NY 11560-1025, USA

52 Partially supported by the International Doctorate on Astroparticle Physics (IDAPP) program

${ }^{53}$ Institució Catalana de Recerca i Estudis Avançats (ICREA), Barcelona, Spain

${ }^{54}$ Consorzio Interuniversitario per la Fisica Spaziale (CIFS), I-10133 Torino, Italy

55 INTEGRAL Science Data Centre, CH-1290 Versoix, Switzerland

56 NASA Postdoctoral Program Fellow, USA
$F(\geq 100 \mathrm{MeV}) \sim(0.22-0.65) \times 10^{-7} \mathrm{~cm}^{-2} \mathrm{~s}^{-1}$ for their "nominal" model. However, attempts to observe such emission with EGRET (1991-1995) yielded only an upper limit of $2.0 \times 10^{-7} \mathrm{~cm}^{-2} \mathrm{~s}^{-1}$ above $100 \mathrm{MeV}$ at $95 \%$ confidence level (Thompson et al. 1997).

The existence of an additional, spatially extended component of the solar emission due to the inverse Compton (IC) scattering of CR electrons off solar photons was not realized until recently (Moskalenko et al. 2006, Orlando \& Strong 2007). While the IC emission is brightest in the region within a few degrees of the Sun (hereafter we refer the angle relative to the Sun as the "elongation angle"), even at larger elongation angles it can be comparable in intensity to the isotropic (presumably extragalactic) $\gamma$-ray background (Abdo et al.2010c). The flux for both components of the CR-induced emission is expected to change over the solar cycle due to the change of the heliospheric flux of the Galactic CRs in anticorrelation with the variations of the solar activity. Observations of the IC emission provide information about CR electron spectra throughout the entire inner heliosphere simultaneously, thus allowing comprehensive studies of the solar modulation of Galactic CRs in this region. For a moderately high - moderately low level of the solar modulation, the integral IC flux for elongation angles $\leq 6^{\circ}$ was predicted (Moskalenko et al. $2006)$ to be $F(\geq 100 \mathrm{MeV}) \sim(2.0-4.3) \times 10^{-7} \mathrm{~cm}^{-2} \mathrm{~s}^{-1}$, respectively, and thus detectable by the EGRET and FermiLAT. A calculation by Orlando \& Strong (2008) gave a similar flux $F(\geq 100 \mathrm{MeV})=2.18 \times 10^{-\tau} \mathrm{cm}^{-2} \mathrm{~s}^{-1}$ for elongation angles $\leq 10^{\circ}$ for the solar maximum conditions.

Reanalysis of the EGRET data by Orlando \& Strong (2008) led to the detection of both predicted components, point-like hadronic emission from the solar disk and extended leptonic emission from IC scattering of CR electrons on solar photons. Their analysis combined all 11 observational periods when the Sun was in the field of view of EGRET between the beginning of the $C G R O$ mission (April 1991) and the end of the fourth observing cycle (October 1995). The average solar activity for this period was moderate, decreasing from its peak in 1990 to the minimum in 1995 . The Orlando \& Strong (2008) analysis yielded fluxes $F(\geq 100 \mathrm{MeV})=(1.8 \pm 1.1) \times 10^{-7}$ $\mathrm{cm}^{-2} \mathrm{~s}^{-1}$ for the disk and $F(\geq 100 \mathrm{MeV})=(3.8 \pm 2.1) \times$ $10^{-7} \mathrm{~cm}^{-2} \mathrm{~s}^{-1}$ for the IC component for elongation angles $\leq 10^{\circ}$, consistent with their estimate of the IC flux for the solar maximum conditions.

The launch of Fermi in 2008 has made observations of the quiet Sun with high statistical significance and on a daily basis possible. During the first two years of the Fermi mission, the solar activity has been extremely low, resulting in a high heliospheric flux of Galactic CRs. Therefore, the CR-induced quiescent $\gamma$-ray emission from the Sun is expected to be near its maximum. Preliminary analysis of the data from the Fermi Large Area Telescope (Fermi-LAT) showed the existence of both point-like and extended components of solar $\gamma$-ray emission (Brigida 2009, Giglietto 2009; Orlando 2009). In this paper, we report on the Fermi-LAT observations of the quiescent Sun during the first 18 months of the science phase of the mission.

\section{DATA SELECTION AND COUNT MAPS}

Fermi was launched on June 11, 2008 into circular Earth orbit with an altitude of $565 \mathrm{~km}$ and inclination of $25.6^{\circ}$, and an orbital period of 96 minutes. The principal instrument on Fermi is the LAT (Atwood et al.2009), a pair-production tele- 
scope with a large effective area $\left(\sim 8000 \mathrm{~cm}^{-2}\right.$ at $\left.1 \mathrm{GeV}\right)$ and field of view $(2.4 \mathrm{sr}$ ), sensitive to $\gamma$-rays between $20 \mathrm{MeV}$ and $>300 \mathrm{GeV}$. After the commissioning phase, devoted to finetuning of the instrument and calibrations, the Fermi-LAT began routine science operations on August 4, 2008. The FermiLAT normally operates in sky-survey mode where the whole sky is observed every 3 hours (or 2 orbits) with an almostuniform exposure on daily time scales.

The energy dependent systematic uncertainties of the effective area of the instrument were evaluated by comparing the efficiencies of analysis cuts for data and simulations of observations of pulsars (Rando 2009). This study revealed a systematic uncertainty of $10 \%$ at $100 \mathrm{MeV}$, decreasing to $5 \%$ at $560 \mathrm{MeV}$, and increasing to $20 \%$ at $10 \mathrm{GeV}$ and above. The photon angular resolution is also energy dependent. The $68 \%$ containment angle averaged over the Fermi-LAT acceptance (the width of the point-spread function - PSF) can be approximated by the following expression: $\left\langle\Theta_{68}(\epsilon)\right\rangle=$ $\left(\left[0.8^{\circ} \epsilon^{-0.8}\right]^{2}+\left[0.07^{\circ}\right]^{2}\right)^{1 / 2}$, where $\epsilon$ is the photon energy in $\mathrm{GeV}$. More details on the instrument performance can be found in the Fermi-LAT calibration paper (Atwood et al. 2009). The analysis presented here uses post-launch P6V3 instrument response functions (IRFs). These take into account pile-up and accidental coincidence effects in the detector subsystems that were not considered in the definition of the prelaunch IRFs.

We use the Fermi-LAT data collected between August 4, 2008, and February 4, 2010. Events $\geq 100 \mathrm{MeV}$ arriving with elongation angles $\theta \leq 20^{\circ}$ (region of interest - ROI) and satisfying the Diffuse class selection (Atwood et al. 2009) are used. To reduce the contamination by the $\gamma$-ray emission coming from CR interactions in the Earth's upper atmosphere our selection is refined by selecting events with zenith angles $<105^{\circ}$. To reduce systematic uncertainties due to the bright diffuse $\gamma$-ray emission from the Galactic plane and a possible spillover due to the broad PSF at low energies, we have also excluded the data taken when the Sun was within $30^{\circ}$ of the plane $\left(\left|b_{\odot}\right| \geq 30^{\circ}\right)$. We further excluded the periods when the Sun was within $20^{\circ}$ of the Moon or any other bright celestial source with the integral flux $F_{1 \mathrm{FGL}} \geq 2 \times 10^{-7} \mathrm{~cm}^{-2}$ $\mathrm{s}^{-1}$ above $100 \mathrm{MeV}$ as selected from the 1FGL Fermi-LAT source catalog (Abdo et al.2010a). These various selections produce a very clean event subsample but at the expense of removing about $93 \%$ of the initial ROI data set as summarized in Table 1

Because the Sun is moving across the sky, the analysis of its emission requires special treatment. Therefore, a dedicated set of tools was developed, not a part of the standard FermiLAT Science Tools package, to deal with moving sources such as the Sun and the Moon. Using these specialized tools, the data are selected in a moving frame centered on the instantaneous solar position, which is computed using an interface to the JPL ephemeris libraries ${ }^{57}$.

\section{ANALYSIS METHOD}

For the analysis of the Sun-centered maps we used the Fermi-LAT Science Tools ${ }^{58}$ version 9r16p0. The gtlike tool provides maximum likelihood parameter values (using the method described in Cash 1979, Strong 1985; Mattox

\footnotetext{
${ }^{57}$ http://iau-comm4.jpl.nasa.gov/access2ephs.html

58 Available from Fermi Science Support Center (FSSC), http://fermi.gsfc.nasa.gov/ssc
}

et al. 1996), which derives error estimates (and a full covariance matrix) from Minuit, a minimization tool supported by CERN, using the quadratic approximation around the best fit.

\subsection{Background determination}

The correct evaluation of the background in the region around the Sun is of considerable importance for the analysis of the weak extended IC emission. The latter is expected to decrease as $\sim 1 / \theta$ with elongation angle $\theta$ (Moskalenko et al. 2006; Orlando \& Strong 2007) and becomes indistinguishable from the background for $\theta \gtrsim 20^{\circ}$. The background is mainly due to the diffuse Galactic and isotropic (presumably extragalactic) $\gamma$-ray emission averaged along the ecliptic and to weak point sources. The evaluation of the background was done by two methods, one based on the analysis of flight data and the other on simulations.

The first method utilizes the data and is called a "fake-Sun" analysis, where an imaginary source trails the Sun along the ecliptic. For this method, application of exactly the same sets of cuts as are used for the Sun yields an estimate of the background. Since the extended solar IC emission is insignificant for elongation angles $\theta \gtrsim 20^{\circ}$ we used $40^{\circ}$ as the minimum trailing distance. To reduce statistical errors in the background determination, the background is averaged over 4 fake-Sun sources displaced from each other and from the Sun itself by $40^{\circ}$ intervals along the ecliptic. Because all fake-Sun sources are sampling the same area on the sky, the backgrounds determined using individual fake-Sun sources are consistent within a fraction of a percent. Note that the 18month analysis period is long enough to average out any effects connected with incomplete sampling of the background. This would be an issue if the analysis period was shorter than 12 months.

Figure 1 shows the Gaussian-smoothed count maps $>100$ $\mathrm{MeV}$ centered on the solar position and the hypothetical trailing source (average of the 4 fake-Suns). The solar emission is clearly seen on the left panel, while the right panel shows the background, which is essentially uniform. The integral intensity distribution for the two samples, centered at the solar position and centered on the averaged fake-Sun source, is shown in Figure 2 The number of events per solid angle is shown vs. the angular distance from the Sun (the elongation angle) and the fake-Sun positions for a bin size $0.25^{\circ}$. While for the solar centered data set the integral intensity increases considerably for small elongation angles, the averaged fakeSun profile is flat. The two distributions overlap at distances larger than $20^{\circ}$ where the signal significance is diminished. The gradual increase in the integral intensity for $\theta \gtrsim 25^{\circ}$ is due to the bright Galactic plane broadened by the PSF, see the event selection cuts summarized in Section 2 and Table 1.

The second method of evaluating the background uses an all-sky simulation which takes into account a model of the diffuse emission (including the Galactic and isotropic components, gll_iem_v02.fits and isotropic_iem_v02.txt, correspondingly; see footnote 58) and the sources from 1FGL FermiLAT catalog (Abdo et al.2010a). To the simulated sample we apply the same set of cuts as applied to the real data and select a subsample centered on the position of the real Sun. The simulated background is then compared with the background derived from a fit to the fake-Sun in the first method. Figure 3 shows the spectra of the background derived by the two methods. The agreement between the two methods (and the spectrum of the diffuse emission at medium and high latitudes, Abdo et al.2010c not shown) is very good, showing that the 
background estimation is well understood and that there is no unaccounted or missing emission component in the analysis.

Finally, we check the spatial uniformity of the background determined by the fake-Sun method. The ROI restricted by $\theta \leq 20^{\circ}$ was divided into nested rings. We use 4 annular rings with radii $\theta=10^{\circ}, 14^{\circ}, 17.3^{\circ}$, and $20^{\circ}$, which were chosen to subtend approximately the same solid angle for each ring, and hence should contain approximately equal numbers of background photons if their distribution is spatially flat. The ring-by-ring background intensity variations were found to be less than $1 \%$. Note that the background emission is considerably more intense than the expected IC component (see Section 3.2, and even small background variations across the ROI may affect the analysis results. To minimize these systematic errors, we therefore using the ring method for the background evaluation.

The evaluated spectrum of the background for $\theta \leq 20^{\circ}$ was fitted using the maximum likelihood method and the results were used to derive the simulated average photon count per pixel using the gtmodel routine from the Fermi-LAT Science Tools. For each pixel of the predicted average photon count distribution, we generate a set of 100 random events assuming Poisson statistics and compare them with the observed number of photons in the corresponding pixel of the map centered on the fake-Sun, as shown in Figure 4. The resulting distribution of the difference of counts between the observed and simulated photons per pixel is consistent with a normal distribution of mean $0.155 \pm 0.002$ and standard deviation $2.337 \pm 0.002$.

\subsection{Separation of the solar emission components}

The separation of the disk and extended components of the solar emission was done in a model-independent way. The Sun-centered maps of Fermi-LAT $\gamma$-ray counts were analyzed using a maximum likelihood technique where the background parameters in each nested ring were fixed using the fake-Sun method as described above. The flux and spectral index of the extended emission were determined independently in each energy range in each nested ring, while the disk component (modeled as the Fermi-LAT PSF because the $\sim 0.5^{\circ}$ solar disk is not resolvable) was allowed to have a free spectral index and flux normalization. The choice of the annular radii $\left(5^{\circ}, 11^{\circ}\right.$, and $\left.20^{\circ}\right)$ has been optimized to have a likelihood test statistic $^{59}$ (TS) value $\geq 25$ for the fitted IC component in each ring.

Figure 5 shows the angular distribution of photons $\geq 500 \mathrm{Me} V$ from the Sun on a $0.2^{\circ}$ grid, the background determined by the fake-Sun method, and the fitted disk and extended components. The spectrum of a disk source is modeled as a power law with the total flux and spectral index obtained from the fit of the disk component. The extended emission is modeled as the sum of the individual fits in each energy bin in each nested ring. The observed angular distribution can be well fitted only by adding an extended IC component.

\section{RESULTS}

The analysis of the Fermi-LAT observations for the first 18 months of the mission gives a significant detection and separation of the two components of solar $\gamma$-ray emission. The large photon statistics allow us to derive the spectral shape of each component by fitting them in narrow energy bands,

\footnotetext{
59 The likelihood test statistic TS is defined in Mattox et al. (1996).
}

so that the exposures and convolutions with the PSF do not depend strongly on a the assumed spectral shape.

Table 2 gives the energy spectrum for the IC component from the model-independent analysis or elongation angles $\leq 5^{\circ}$, which corresponds to the radius of the innermost ring, and $\leq 20^{\circ}$. The fitted integral fluxes for the IC component in each ring are given in Table 3 together with model calculations (described in Section 5). The energy bin size was selected to provide good convergence of the likelihood fit in each energy interval. The observed spectrum for the disk component is given in Table 4

Evaluation of the systematic errors for each component of the solar emission has to take into account the uncertainties in the effective area as a function of energy as indicated in Section 2 and the statistical uncertainties in the determination of the background (Section 3.1). The uncertainties associated with the effective area were propagated using the modified effective areas bracketing the nominal ones (P6V3 Diffuse) (Abdo et al. 2010b). The uncertainties in the background were taken into account by assigning $\pm 1 \sigma$ deviation to the background flux and repeating the fit for all components.

\section{CALCULATION OF THE IC EMISSION}

The calculation of the IC emission from the solar radiation field was first described by Moskalenko et al. (2006) and Orlando \& Strong (2008) using the formula for the differential interaction rate for an anisotropic distribution of target photons (Moskalenko \& Strong 2000). The CR electron distribution was assumed to be isotropic (hereafter we use the term electrons to refer to both electrons and positrons). The upscattering of optical solar photons to the Fermi-LAT energy range $\geq 100 \mathrm{MeV}$ involves $\mathrm{CR}$ electrons above $\sim 2 \mathrm{GeV}$. At energies below $\sim 20 \mathrm{GeV}, \mathrm{CR}$ electrons in the heliosphere are subject to significant adiabatic energy losses and drifts in the magnetic field: the combined effect is called the heliospheric modulation (e.g., Potgieter 1998). A calculation of the spatially extended emission due to the IC scattering of CR electrons on solar photons requires the integration of $\gamma$-ray yields along the line-of-sight folded with the CR electron spectrum at different heliospheric distances (Moskalenko et al. 2006).

Due to the radial distribution of the solar photons the IC emission is brightest at small elongation angles, and is distributed over the whole sky at low levels. The differential flux of upscattered photons depends on how the CR electron spectrum changes with heliocentric distance, which is the only unknown in the calculations. Therefore, observations of the IC component of the solar emission provide a new tool to probe the CR electron spectrum in the inner heliosphere $(r<10$ $\mathrm{AU})$ down to close proximity to the Sun.

\subsection{CR modulation in the heliosphere}

Studies of the CR modulation are based on the solution of the Parker (1965) transport equation (e.g., see reviews by Potgieter 1998, Heber et al. 2006). Particle transport to the inner heliosphere is mainly determined by spatial diffusion, convection by the solar wind, and adiabatic cooling. Besides, rotation of the Sun causes the interplanetary magnetic field in the solar equatorial plane to be distinctly different from the field above and below the poles, the so-called Parker's spiral (Parker 1958). This, in turn, causes differences in the spectra of CR particles in these regions. Realistic timedependent three-dimensional hydrodynamic models incorporating these effects have been developed (e.g., Florinski et al. 
2003, Langner et al. 2006, Potgieter \& Langner 2004); however, the effect of heliospheric modulation is still far from being fully understood. One of the major difficulties in developing models of heliospheric modulation is that the data gathered by spacecraft taken at different heliospheric distances are often at different energies and related to different modulation levels. The region $<1 \mathrm{AU}$ is the least studied. Another problem is that the input information for the modulation models, such as the local interstellar spectra of CR species, is missing.

Although much effort has gone into development of realistic modulation models, the method most often used is the socalled "force-field" approximation (Gleeson \& Axford 1968), which employs a single parameter - the "modulation potential" $\Phi$ - that varies over the solar cycle to characterize the strength of the modulation effect on the CR spectra:

$$
J_{e}(r, E)=J_{e}(\infty, E+\Phi[r]) \frac{\left(E^{2}-m_{e}^{2} c^{4}\right)}{\left(\{E+\Phi[r]\}^{2}-m_{e}^{2} c^{4}\right)},
$$

where $J_{e}(\infty, E+\Phi[r])$ is the local interstellar electron spectrum, $E$ is the total electron energy, $m_{e} c^{2}$ is the electron rest mass, $\Phi(r)$ is the modulation potential, and $r$ is the distance from the Sun. Even though this approximation is very crude and implies spherical symmetry for the heliosphere, it can be used as a convenient parameterization of the CR spectrum at different levels of solar activity.

Expressions for $\Phi(r)$ were derived by Moskalenko et al. (2006) based on the radial dependence of the CR mean free path given by Fujii \& McDonald (2005) for Cycles 20/22 and and for Cycle 21 assuming separability of the heliospheric diffusion coefficient into radial and energy-dependent functions. For Cycles 20/22, Moskalenko et al. (2006) obtain:

$$
\Phi_{1}(r)=\frac{\Phi_{0}}{1.88} \begin{cases}r^{-0.4}-r_{b}^{-0.4}, & r \geq r_{0}, \\ 0.24+8\left(r^{-0.1}-r_{0}^{-0.1}\right), & r<r_{0},\end{cases}
$$

where $\Phi_{0}$ is the modulation potential at $1 \mathrm{AU}, r_{0}=10 \mathrm{AU}$, and $r_{b}=100 \mathrm{AU}$ is the heliospheric boundary. For Cycle 21 they give:

$$
\Phi_{2}(r)=\Phi_{0}\left(r^{-0.1}-r_{b}^{-0.1}\right) /\left(1-r_{b}^{-0.1}\right) .
$$

These formulae were derived for $r \gtrsim 1$ AU. Closer to the Sun at $0.3 \mathrm{AU} \lesssim r<1 \mathrm{AU}$ there are only very few measurements of Galactic CR protons and helium, and these are at low energies $\lesssim 60 \mathrm{MeV}$ nucleon ${ }^{-1}$ (e.g., Christon et al. 1975, Kunow et al. 1977, Müller-Mellin et al. 1977). Since CR transport is strongly energy dependent, these low-energy protons and helium nuclei measurements are irrelevant to the current study and CR transport is very uncertain. Therefore, as a first approximation we use eqs. (2), (3) for the entire heliosphere (Models 1, 2), from the solar surface to the heliospheric boundary $R_{\odot}<r<r_{b}$, except for Model 3 described below.

\subsection{The CR electron spectrum}

Assuming that the $\mathrm{CR}$ propagation in the heliosphere is spherically symmetric in the first approximation, the CR electron spectrum at different heliospheric distances is the only unknown in evaluating the solar IC flux. Comparison of the model calculations with observations provides a method to probe the CR electron spectra at different heliospheric distances $r \lesssim 10$ AU. Because of the lack of CR measurements outside of the heliosphere, we have to rely on a comprehensive Galactic CR propagation model tuned to other CR data and diffuse $\gamma$-ray emission, such as GALPROP (Strong \& Moskalenko 1998; Moskalenko \& Strong 1998; Ptuskin et al.
2006; Abdo et al. 2009a, 2010c; Vladimirov et al. 2010), or measurements of the flux at $1 \mathrm{AU}$.

In this paper we use the $\mathrm{CR}$ electron spectrum recently measured by the Fermi-LAT (Abdo et al. 2009b, Ackermann et al. 2010) between $7 \mathrm{GeV}$ and $\sim 1 \mathrm{TeV}$. At energies $<7 \mathrm{GeV}$ we use the Alpha Magnetic Spectrometer (AMS-01) lepton data (Alcaraz et al. 2000) collected in its flight in June 1998, which are the most accurate to date, but were made in the previous solar cycle. Preliminary results by the Payload for Antimatter Matter Exploration and Light-nuclei Astrophysics (PAMELA, Adriani et al.2011), in orbit since June 2006, indicate that the electron spectrum $>7 \mathrm{GeV}$ is consistent with the Fermi-LAT data, but is more intense than the AMS-01 data at lower energies.

Unusually high intensities of Galactic CRs during the period of anomalously low solar activity were also reported by the Advanced Composition Explorer (ACE), which monitors the flux of CR species at $1 \mathrm{AU}$ in a few $100 \mathrm{MeV} /$ nucleon energy range. The measured intensities of major species from $\mathrm{C}$ to $\mathrm{Fe}$ (Mewaldt et al. 2010) were each 20\%-26\% greater in late 2009 than in the 1997-1998 minimum and previous solar minima of the space age (1957-1997). While the value of $\Phi_{0}$ is somewhat arbitrary and depends on the assumed local interstellar spectrum (but does not depend on the particle type), the unusually low solar activity during the observational period suggests that the modulation of Galactic CRs should be considerably weaker than at any other time.

Therefore, the Fermi-LAT CR electron spectrum was fitted using a parameterization for the local interstellar spectrum and assuming a relatively low value of the modulation potential $\Phi_{0}=\Phi(1 \mathrm{AU})=400 \mathrm{MV}$ (eq. [1]):

$$
J_{e}(\infty, E)=\left\{\begin{array}{l}
a(b+c)^{-d}(E / b)^{-3}, E<b, \\
a(E+c)^{-d}, E \geq b,
\end{array}\right.
$$

where $J_{e}$ is in units $\mathrm{m}^{-2} \mathrm{~s}^{-1} \mathrm{sr}^{-1} \mathrm{GeV}^{-1}, a=160.24 \mathrm{~m}^{-2}$ $\mathrm{s}^{-1} \mathrm{sr}^{-1} \mathrm{GeV}^{2.03}, b=7 \mathrm{GeV}, c=-1.20 \mathrm{GeV}, d=3.03$, and $E$ is the total electron energy in GeV. Different values for $\Phi_{0}$ yield somewhat different values for the other parameters while providing the same fit to the Fermi-LAT electron spectrum. We found that the value of $\Phi_{0}$ given above provides reasonable agreement with the observed IC flux (see Section 4); more detailed analysis of the electron spectrum in the inner heliosphere based on Fermi-LAT observations of the IC emission will be given in a forthcoming paper.

Since most of the IC emission is produced by electrons in the inner heliosphere $r<10 \mathrm{AU}$, eqs. (1), (4) is a good approximation of the $\mathrm{CR}$ electron spectrum even though it may produce an unphysical spectrum beyond the heliospheric boundary at $r_{b}=100 \mathrm{AU}$. (In fact, for elongation angles $\theta \leq 20^{\circ}$ most of the emission is produced by electrons at $r \lesssim 2$ AU, see Figure 2 in Moskalenko et al. 2006.) However, the electron spectrum obtained in such a way is also close to the local interstellar spectrum that is calculated by GALPROP and used for modeling of the Galactic diffuse $\gamma$-ray emission (Abdo et al. 2009a, 2010c).

Using this parameterization for the CR electron spectrum, we construct three models to compare with the data. To obtain the electron spectrum at an arbitrary heliospheric distance, $r$, Models 1 and 2 use parameterizations $\Phi_{1,2}(r)$ (with $\Phi_{0}=$ $400 \mathrm{MV}$ ) given by eqs. (2), (3), respectively. Model 3 uses parameterization eq. (2) as specified above, but assuming no additional modulation for $r<1 \mathrm{AU}$, i.e., $\Phi(r<1 \mathrm{AU})=$ $\Phi_{0}$. In this model the electron spectrum at $<1 \mathrm{AU}$ is the same as measured by the Fermi-LAT, which provides us with an 
upper limit for CR electron flux closer to the Sun.

Figure 6 shows the fit to the Fermi-LAT electron spectrum. The AMS-01 lepton data were collected during the period of rising solar activity ${ }^{60}$, where the low modulation potential adapted for the current solar minimum is not appropriate. These data are not fitted and shown for only illustration. To illustrate the effect of different parameterizations $\Phi_{1,2}(r)$, the Figure also shows the spectra for Models 1,2 at $r=0.3$ AU. All three models of the electron spectrum are normalized to the same Fermi-LAT data at 1 AU. Therefore the largest difference between the models is at low energies where the modulation effect is strongest.

\section{EMISSION FROM THE SOLAR DISK}

A detailed theoretical study of $\gamma$-ray emission from the solar disk was published by Seckel et al. (1991). The predicted flux is very sensitive to the assumptions about CR transport in the inner heliosphere, the magnetic fields in the solar atmosphere, and the CR cascade development in the solar atmosphere.

The Seckel et al. (1991) calculation divides space into three regions: the interplanetary space, the corona, and the regions below the corona. The interplanetary magnetic field $\left(>2 R_{\odot}\right)$ is taken to be in the form of a Parker (1958) spiral with the total field $\sim 50 \mu \mathrm{G}$ near the Earth. Inferior to Earth's orbit the field is nearly radial at angle $\sim 45^{\circ}$. CR propagation from the Earth's orbit to the bottom of the corona is treated as spherically symmetrical diffusion with no absorption (i.e., neglecting CR interactions). The adopted diffusion coefficient has a linear dependence on energy, while the spatial radial dependence is a power-law with index 2 . The CR spectrum at 1 AU was adopted from Webber \& Potgieter (1989), which is a parameterization of the measurements made during balloon flights in 1976 and 1979 (Webber et al. 1987).

Interior to the corona, the CR propagation and absorption (interactions) are treated simultaneously using a Monte Carlo code. The magnetic field configuration is chosen corresponding to a quiet Sun (Priest 1982), where the average field strengths are of the order of a few Gauss. However, the fields are non-uniform with the flux bundles $\left(\sim 10^{3} \mathrm{G}\right.$, a few hundred kilometers across) located at the corners of convective cells and separated by thousands of kilometers. The chromosphere is assumed to be isothermal in hydrostatic equilibrium which gives an exponential density profile with scale height $\sim 115 \mathrm{~km}$. The density profile below the photosphere is taken from Baker \& Temesvary (1966). The characteristic column density is $\sim 40 \mathrm{~g} \mathrm{~cm}^{-3}$ at $500 \mathrm{~km}$ depth and $100 \mathrm{~g} \mathrm{~cm}^{-3}$ at $900 \mathrm{~km}$.

The cascades initiated by high-energy particles $(>3 \mathrm{TeV})$ do not contribute much to the observed $\gamma$-ray flux and are neglected. The solar magnetic fields do not significantly affect their directionality until particle energies drop below $\sim 10$ $\mathrm{GeV}$ - by that time most cascades are deep enough so that only a few low-energy photons will escape. On the other hand, the low-energy primaries produce cascades for which a significant amount of energy is reflected back to the solar surface (so-called mirrored showers). The typical low-energy cascade has less than a few interaction lengths of material to pass through before photons can escape. Therefore, such cascades act as if they evolve in moderately thin targets.

${ }^{60}$ See the charts of the computed tilt angle of the heliospheric current sheet at http://wso.stanford.edu/
The actual yields are calculated by propagation of onedimensional cascades through a slab. The photon yield includes only the photons that make it through the slab. Secondary electrons, positrons, and baryons exiting the slab are ignored even though they are likely to reenter the Sun. This may underestimate the actual $\gamma$-ray flux.

The Seckel et al. (1991) calculations were made in two scenarios: (i) neglecting the effects of interplanetary magnetic field on particle propagation and assuming the solar surface is fully absorbing (so-called "naive" model), and (ii) the "nominal" model, which includes all the assumptions about CR diffusion in the inner heliosphere and corona. The integral flux above $100 \mathrm{MeV}$ was predicted to be $F(\geq 100 \mathrm{MeV}) \sim$ $(0.22-0.65) \times 10^{-7} \mathrm{~cm}^{-2} \mathrm{~s}^{-1}$ for the "nominal" model, where the range corresponds to the different assumptions about the CR cascade development: slant vs. more realistic mirrored showers (i.e., reflected back to the solar surface).

\section{DISCUSSION AND CONCLUSIONS}

Figures 7 and 8 show the results of the analysis of the extended emission component together with the model predictions (Section 5). The plotted values are obtained from the IC flux in each ring as shown in Table 3 divided by the corresponding solid angle. The model calculations are shown unbinned (curves) and binned with the same bin size as used for the data. Although the highest energy point $3-10 \mathrm{GeV}$ shows some excess relative to the model predictions, this is difficult to explain from the model viewpoint since the effect of the solar modulation is decreasing at high energies thus making the model more accurate. Future analysis with larger statistics should clarify if this discrepancy is real. The agreement of the observed spectrum and the angular profile of the IC emission with the model predictions (as described in Section 5p below a few $\mathrm{GeV}$ is very good. The innermost ring used for the analysis of the IC emission subtends an angular radius of $5^{\circ}$ corresponding to a distance $\sim 0.1 \mathrm{AU}$ from the Sun, i.e. 4 times closer to the Sun than Mercury. At such a close proximity to the Sun, and actually anywhere $<1 \mathrm{AU}$, the spectrum of CR electrons has never been measured.

It does not seem possible to discriminate between the models at the current stage. The spectral shape $<1 \mathrm{GeV}$ in Figure 7 and the intensity in the innermost ring in Figure 8 is better reproduced by Models 1, 2, while the intensity in the middle ring $5^{\circ}-11^{\circ}$ (Figure 8 ) is better reproduced by Model 3 . Even though the current data do not allow us to discriminate between different models of the CR electron spectrum at close proximity to the Sun, the described analysis demonstrates how the method would work once the data become more accurate. In particular, it is possible to increase the statistics by four-fold by masking out the background sources or modeling them, instead of requiring the angular separation between bright sources and the Sun to be $>20^{\circ}$ (Table 1 ). More details will be given in a forthcoming paper. The increase of the solar activity may also present a better opportunity to distinguish between the models since the difference between the model spectra of CR electrons will increase with solar modulation.

The intensity of the IC component is comparable to the intensity of the isotropic $\gamma$-ray background even for relatively large elongation angles (Table 2). Integrated for subtended angles $\leq 5^{\circ}$, the latter yields $\sim 2.5 \times 10^{-7} \mathrm{~cm}^{-2} \mathrm{~s}^{-1}$ above $100 \mathrm{MeV}$ (Abdo et al. 2010c) vs. $\sim 1.4 \times 10^{-7} \mathrm{~cm}^{-2} \mathrm{~s}^{-1}$ for the IC component. For subtended angles $\leq 20^{\circ}$, the integral flux of the isotropic $\gamma$-ray background is $\sim 3.9 \times 10^{-6} \mathrm{~cm}^{-2}$ 
$\mathrm{s}^{-1}$ above $100 \mathrm{MeV}$ vs. $\sim 6.8 \times 10^{-7} \mathrm{~cm}^{-2} \mathrm{~s}^{-1}$ for the IC component. Therefore, it is important to take into account the broad nonuniform IC component of the solar emission when dealing with weak sources near the ecliptic. The relative importance of the IC component will increase with time since the upper limit on the truly diffuse extragalactic emission could be lowered in future as more $\gamma$-ray sources are discovered and removed from the analysis.

Figure 9 shows the spectrum for the disk component measured by the Fermi-LAT (Table 4) and two model predictions ("naive" and "nominal") by Seckel et al. (1991) as described in Section 6 In each set of curves, the lower bound (dotted line) is the CR induced $\gamma$-ray flux for the slant depth model, and the upper bound (solid line) is the $\gamma$-ray flux assuming showers are mirrored (as charged particles would be). The observed spectrum can be well fitted by a single power-law with a spectral index of $2.11 \pm 0.73$. The integral flux of the disk component is about a factor of 7 higher than predicted by the "nominal" model. An obvious reason for the discrepancy could be the conditions of the unusually deep solar minimum during the reported observations. However, this alone can not account for such a large factor, see a comparison with the EGRET data below. Another possibility for an estimated "nominal" flux to be so low compared to the Fermi-LAT observations is that the secondary particles produced by CR cascades exiting the atmospheric slab are ignored in the calculation while they are likely to reenter the Sun. On the other hand, the proton spectrum by Webber et al. (1987) used in the calculation is about a factor of 1.5 higher above $\sim 6 \mathrm{GeV}$ than that measured by the BESS experiment in 1998 (see Figure 4 in Sanuki et al. 2000). Meanwhile, calculation of the disk emission relies on assumptions about CR transport in the inner heliosphere and in the immediate vicinity of the Sun thus allowing for a broad range of models (cf. "naive" vs. "nominal" models). The accurate measurements of the disk spectrum by the Fermi-LAT thus warrant a new evaluation of the $\mathrm{CR}$ cascade development in the solar atmosphere.

The spectral shape of the observed disk spectrum is close to the predictions except below $\sim 230 \mathrm{MeV}$ where the predicted spectral flattening is not confirmed by the observations. This may be due to the broad PSF making it difficult to distinguish between the components of the emission or a larger systematic error below $\sim 200 \mathrm{MeV}$ associated with the IRFs.

The results of Fermi-LAT observations can be also compared with those from the analysis of the EGRET data (Orlando \& Strong 2008). The latter gives an integral flux $(\geq 100 \mathrm{MeV})$ for the disk component of $(1.8 \pm 1.1) \times 10^{-7}$ $\mathrm{cm}^{-2} \mathrm{~s}^{-1}$; for the IC flux Orlando \& Strong (2008) obtain $(3.9 \pm 2.2) \times 10^{-7} \mathrm{~cm}^{-2} \mathrm{~s}^{-1}$ for elongation angles $\leq 10^{\circ}$. The EGRET-derived integral IC flux is very close to the flux observed by the Fermi-LAT for the same range of elongation angles (Table 3). Meanwhile, the integral flux of the disk component observed by the Fermi-LAT (Table 4) is a factor of 2.5 higher than obtained from EGRET data. Such an increase in the $\gamma$-ray flux from the solar disk may imply a significant variation of the disk emission over the solar cycle. This is not surprising since the disk flux depends on the ambient $\mathrm{CR}$ proton spectrum in the immediate proximity of the solar atmosphere, in contrast to the solar IC emission, which is produced by electrons in a considerable part of the heliosphere and integrated along the line of sight. However, such variations should be confirmed by future observations.

The analysis reported here refers to the period of the so- lar minimum. However, the solar activity is just starting to pick up for the current cycle. We expect that the effects of the increased solar activity on the fluxes of the two components of the emission will be different and it is important to follow their evolution during the whole solar cycle. Continuous monitoring of the solar emission by the Fermi-LAT over the whole solar cycle will enable us to study CR transport in the inner heliosphere, to improve models of the solar modulation, and of the development of CR cascades in the solar atmosphere.

The Fermi-LAT Collaboration acknowledges generous ongoing support from a number of agencies and institutes that have supported both the development and the operation of the Fermi-LAT as well as scientific data analysis. These include the National Aeronautics and Space Administration and the Department of Energy in the United States, the Commissariat à l'Energie Atomique and the Centre National de la Recherche Scientifique/Institut National de Physique Nucléaire et de Physique des Particules in France, the Agenzia Spaziale Italiana and the Istituto Nazionale di Fisica Nucleare in Italy, the Ministry of Education, Culture, Sports, Science and Technology (MEXT), High Energy Accelerator Research Organization (KEK) and Japan Aerospace Exploration Agency (JAXA) in Japan, and the K. A. Wallenberg Foundation, the Swedish Research Council and the Swedish National Space Board in Sweden. Additional support for science analysis during the operations phase is gratefully acknowledged from the Istituto Nazionale di Astrofisica in Italy and the Centre National d'Études Spatiales in France. I. V. M. and E. O. acknowledge support from NASA grant NNX10AD12G.

\section{REFERENCES}

Abdo, A. A., et al. 2009a, Physical Review Letters, 103, 251101

-. 2009b, Physical Review Letters, 102, 181101

-. 2010a, ApJS, 188, 405

-. 2010b, ApJ, 708, 1254

—. 2010c, Physical Review Letters, 104, 101101

Ackermann, M., et al. 2010, Phys. Rev. D, 82, 092004

Adriani, O., et al. 2011, ArXiv: 1103.2880

Alcaraz, J., et al. 2000, Physics Letters B, 484, 10

Atwood, W. B., et al. 2009, ApJ, 697, 1071

Baker, N., \& Temesvary, S. 1966, Tables of Convective Stellar

Atmospheres, 2nd edition (NASA Institute for Space Studies, New York)

Brigida, M. 2009, in The XLIVth Rencontres de Moriond, "Very High

Energy Phenomena in the Universe", 115

Cash, W. 1979, ApJ, 228, 939

Christon, S., Daly, S., Eraker, J. H., Lamport, J. E., Lentz, G., \& Simpson, J. A. 1975, in Proc. 14th Int. Cosmic Ray Conf. (München), Vol. 5, 1848-+

Chupp, E. L., Forrest, D. J., Higbie, P. R., Suri, A. N., Tsai, C., \& Dunphy, P. P. 1973, Nature, 241, 333

Dolan, J. F., \& Fazio, G. G. 1965, Reviews of Geophysics, 3, 319

Florinski, V., Zank, G. P., \& Pogorelov, N. V. 2003, Journal of Geophysical Research (Space Physics), 108, 1228

Fujii, Z., \& McDonald, F. B. 2005, Advances in Space Research, 35, 611

Giglietto, N. 2009, in American Institute of Physics Conference Series, Vol.

1112, American Institute of Physics Conference Series, ed. D. Bastieri \& R. Rando, 238-241

Gleeson, L. J., \& Axford, W. I. 1968, ApJ, 154, 1011

Heber, B., Fichtner, H., \& Scherer, K. 2006, Space Science Reviews, 125, 81

Hudson, H. S. 1989, in Gamma Ray Observatory Science Workshop, ed. W. N. Johnson, 4-351

Kanbach, G., Bertsch, D. L., Fichtel, C. E., Hartman, R. C., Hunter, S. D., Kniffen, D. A., Kwok, P. W., Lin, Y. C., Mattox, J. R., \&

Mayer-Hasselwander, H. A. 1993, A\&AS, 97, 349

Kunow, H., Wibberenz, G., Green, G., Mueller-Mellin, R., Witte, M.,

Hempe, H., Mewaldt, R. A., Stone, E. C., \& Vogt, R. E. 1977, in Proc. 15th Int. Cosmic Ray Conf. (Plovdiv), Vol. 3, 227-232

Langner, U. W., Potgieter, M. S., Fichtner, H., \& Borrmann, T. 2006, ApJ, 640,1119

Mattox, J. R., et al. 1996, ApJ, 461, 396

Mewaldt, R. A., et al. 2010, ApJ, 723, L1 
Abdo et al.

Moskalenko, I. V., Porter, T. A., \& Digel, S. W. 2006, ApJ, 652, L65

Moskalenko, I. V., \& Strong, A. W. 1998, ApJ, 493, 694

-. 2000, ApJ, 528, 357

Müller-Mellin, R., Witte, M., Hempe, H., Kunow, H., Wibberenz, G., \&

Green, G. 1977, in Proc. 15th Int. Cosmic Ray Conf. (Plovdiv), Vol. 3, 226-+

Orlando, E. 2009, ArXiv: 0907.0557

Orlando, E., \& Strong, A. W. 2007, Ap\&SS, 309, 359

-. 2008, A\&A, 480, 847

Parker, E. N. 1958, ApJ, 128, 664

-. 1965, Planet. Space Sci., 13, 9

Peterson, L. E., Schwartz, D. A., Pelling, R. M., \& McKenzie, D. 1966, J. Geophys. Res., 71, 5778

Peterson, L. E., \& Winckler, J. R. 1959, J. Geophys. Res., 64, 697

Potgieter, M., \& Langner, U. 2004, Annales Geophysicae, 22, 3729

Potgieter, M. S. 1998, Space Science Reviews, 83, 147

Priest, E. R. 1982, Geophysics and Astrophysics Monographs, 21

Ptuskin, V. S., Moskalenko, I. V., Jones, F. C., Strong, A. W., \& Zirakashvili, V. N. 2006, ApJ, 642, 902

Rando, R. 2009, ArXiv: 0907.0626

Sanuki, T., et al. 2000, ApJ, 545, 1135

Seckel, D., Stanev, T., \& Gaisser, T. K. 1991, ApJ, 382, 652

Strong, A. W. 1985, A\&A, 150, 273

Strong, A. W., \& Moskalenko, I. V. 1998, ApJ, 509, 212

Thompson, D. J., Bertsch, D. L., Morris, D. J., \& Mukherjee, R. 1997, J. Geophys. Res., 102, 14735

Vladimirov, A. E., et al. 2010, ArXiv: 1008.3642

Webber, W. R., Golden, R. L., \& Stephens, S. A. 1987, in International Cosmic Ray Conference, Vol. 1, 325-328

Webber, W. R., \& Potgieter, M. S. 1989, ApJ, 344, 779 
Table 1

Summary of the event selection cuts

\begin{tabular}{lcc}
\hline \hline Cumulative event selections & $\begin{array}{c}\text { Photons, } \\
\%\end{array}$ & $\begin{array}{c}\text { Livetime, } \\
\%\end{array}$ \\
\hline$\theta_{\mathrm{ROI}} \leq 20^{\circ}$ & 100 & 100 \\
$\left|b_{\odot}\right| \geq 30^{\circ}$ & 29.2 & 60.9 \\
$\theta_{\mathrm{Moon}}>20^{\circ}$ & 26.2 & 54.8 \\
$\theta\left(F_{1 \mathrm{FGL}}>2 \times 10^{-7} \mathrm{~cm}^{-2} \mathrm{~s}^{-1}\right)>20^{\circ}$ & 6.5 & 17.6 \\
\hline
\end{tabular}

Table 2

Differential spectrum of the IC component

\begin{tabular}{ccccc}
\hline \hline $\begin{array}{c}\text { Energy range, } \\
10^{3} \mathrm{MeV}\end{array}$ & \multicolumn{2}{c}{ Flux \pm stat \pm syst, $\mathrm{cm}^{-2} \mathrm{~s}^{-1} \mathrm{MeV}^{-1}$} & \multicolumn{2}{c}{$\left(E^{2}\right.$ Flux $) \pm \mathrm{stat} \pm \mathrm{syst}, 10^{-5} \mathrm{~cm}^{-2} \mathrm{~s}^{-1} \mathrm{MeV}^{\circ}$} \\
\cline { 2 - 5 } $0.1-0.3$ & $(4.4 \pm 0.9 \pm 0.1) \times 10^{-10}$ & $(2.1 \pm 0.5 \pm 0.3) \times 10^{-9}$ & $1.3 \pm 0.2 \pm 0.03$ & $6.2 \pm 1.4 \pm 0.1$ \\
$0.3-1.0$ & $(4.9 \pm 1.0 \pm 0.1) \times 10^{-11}$ & $(2.2 \pm 0.5 \pm 0.3) \times 10^{-10}$ & $1.4 \pm 0.3 \pm 0.03$ & $6.1 \pm 1.5 \pm 0.9$ \\
$1.0-3.0$ & $(5.5 \pm 1.1 \pm 0.1) \times 10^{-12}$ & $(2.2 \pm 0.6 \pm 0.2) \times 10^{-11}$ & $1.6 \pm 0.3 \pm 0.03$ & $6.1 \pm 1.8 \pm 0.6$ \\
$3.0-10$. & $(6.2 \pm 1.2 \pm 0.1) \times 10^{-13}$ & $(2.2 \pm 0.8 \pm 0.2) \times 10^{-12}$ & $1.9 \pm 0.4 \pm 0.03$ & $6.1 \pm 2.4 \pm 0.6$ \\
& Integral flux \pm stat \pm syst, $\mathrm{cm}^{-2} \mathrm{~s}^{-1}$ & & \\
$\geq 0.1$ & $\left(1.4 \pm 0.2_{-0.4}^{+0.5}\right) \times 10^{-7}$ & $\left(6.8 \pm 0.7_{-0.4}^{+0.5}\right) \times 10^{-7}$ & & \\
\hline
\end{tabular}

Note. - The $E^{2}$ Flux has been evaluated at the geometric mean of each bin.

Table 3

Integral flux $(\geq 100 \mathrm{MeV})$ of the IC component

\begin{tabular}{cccccc}
\hline \hline \multirow{2}{*}{$\begin{array}{c}\text { Elongation } \\
\text { angle }\end{array}$} & TS & \multicolumn{4}{c}{ Integral flux, $10^{-7} \mathrm{~cm}^{-2} \mathrm{~s}^{-1}$} \\
\cline { 3 - 6 } & Flux \pm stat \pm syst & Model 1 & Model 2 & Model 3 \\
\hline $0^{\circ}-5^{\circ}$ & 93 & $1.4 \pm 0.2_{-0.4}^{+0.5}$ & 1.15 & 1.33 & 1.78 \\
$5^{\circ}-11^{\circ}$ & 94 & $2.5 \pm 0.3_{-0.4}^{+0.1}$ & 1.84 & 1.97 & 2.29 \\
$11^{\circ}-20^{\circ}$ & 43 & $3.0 \pm 0.5_{-0.2}^{+0.2}$ & 3.11 & 3.19 & 3.50 \\
$0^{\circ}-10^{\circ}$ & 215 & $3.7 \pm 0.4_{-0.4}^{+0.5}$ & 2.67 & 2.95 & 3.69 \\
$0^{\circ}-20^{\circ}$ & 259 & $6.8 \pm 0.7_{-0.4}^{+0.5}$ & 6.10 & 6.48 & 7.57 \\
\hline
\end{tabular}

Table 4

Differential spectrum of the disk component

\begin{tabular}{cccc}
\hline \hline $\begin{array}{c}\text { Energy range, } \\
10^{3} \mathrm{MeV}\end{array}$ & $\mathrm{TS}$ & $\begin{array}{c}\text { Flux } \pm \text { stat } \pm \text { syst, } \\
\mathrm{cm}^{-2} \mathrm{~s}^{-1} \mathrm{MeV}^{-1}\end{array}$ & $\begin{array}{c}\left(E^{2} \text { Flux }\right) \pm \text { stat } \pm \text { syst, } \\
10^{-5} \mathrm{~cm}^{-2} \mathrm{~s}^{-1} \mathrm{MeV}\end{array}$ \\
\hline $0.10-0.14$ & 59 & $(3.1 \pm 0.5 \pm 0.6) \times 10^{-9}$ & $4.3 \pm 0.7 \pm 0.9$ \\
$0.14-0.19$ & 68 & $(2.4 \pm 0.3 \pm 0.5) \times 10^{-9}$ & $6.1 \pm 0.8 \pm 1.3$ \\
$0.19-0.27$ & 85 & $(7.9 \pm 1.1 \pm 1.6) \times 10^{-10}$ & $4.2 \pm 0.6 \pm 0.8$ \\
$0.27-0.37$ & 100 & $(3.2 \pm 0.4 \pm 0.6) \times 10^{-10}$ & $3.3 \pm 0.4 \pm 0.6$ \\
$0.37-0.52$ & 222 & $(2.2 \pm 0.2 \pm 0.4) \times 10^{-10}$ & $4.2 \pm 0.4 \pm 0.8$ \\
$0.52-0.72$ & 288 & $(1.2 \pm 0.1 \pm 0.2) \times 10^{-10}$ & $4.5 \pm 0.4 \pm 0.7$ \\
$0.72-1.00$ & 243 & $(5.2 \pm 0.6 \pm 1.0) \times 10^{-11}$ & $3.8 \pm 0.4 \pm 0.7$ \\
$1.00-1.39$ & 282 & $(2.8 \pm 0.3 \pm 0.6) \times 10^{-11}$ & $3.9 \pm 0.4 \pm 0.8$ \\
$1.39-1.93$ & 295 & $(1.6 \pm 0.2 \pm 0.3) \times 10^{-11}$ & $4.3 \pm 0.5 \pm 0.8$ \\
$1.93-2.68$ & 193 & $(7.5 \pm 1.1 \pm 1.5) \times 10^{-12}$ & $3.9 \pm 0.6 \pm 0.8$ \\
$2.68-3.73$ & 167 & $(3.4 \pm 0.6 \pm 0.7) \times 10^{-12}$ & $3.4 \pm 0.6 \pm 0.7$ \\
$3.73-5.18$ & 143 & $(2.0 \pm 0.4 \pm 0.4) \times 10^{-12}$ & $3.9 \pm 0.8 \pm 0.8$ \\
$5.18-7.20$ & 85 & $(8.2 \pm 2.1 \pm 1.6) \times 10^{-13}$ & $3.1 \pm 0.8 \pm 0.6$ \\
$7.20-10.0$ & 21 & $(2.2 \pm 0.9 \pm 0.4) \times 10^{-13}$ & $1.6 \pm 0.6 \pm 0.3$ \\
\multicolumn{5}{c}{ Integral flux \pm stat \pm syst, $\mathrm{cm}^{-2} \mathrm{~s}-1$} & \\
$\geq 0.1$ & $\left(4.6 \pm 0.2_{-0.8}^{+1.0}\right) \times 10^{-7}$ & \\
\hline
\end{tabular}

Note. - The $E^{2}$ Flux has been evaluated at the geometric mean of each bin. 


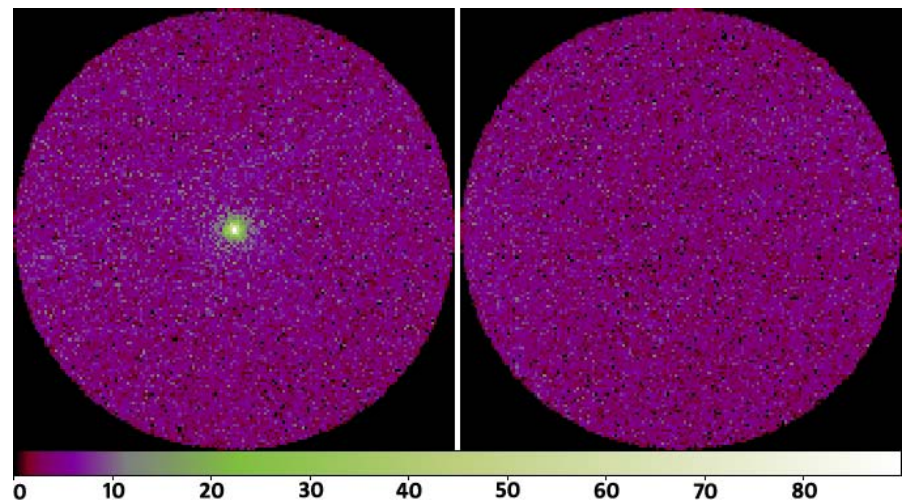

Figure 1. Count maps for events $\geq 100 \mathrm{MeV}$ taken between August 2008 and February 2010 and centered on the Sun (left) and on the trailing source (so-called fake-Sun, right) representing the background. The ROI has $\theta=20^{\circ}$ radius and pixel size $0.25^{\circ} \times 0.25^{\circ}$. The color bar shows the number of counts per pixel.

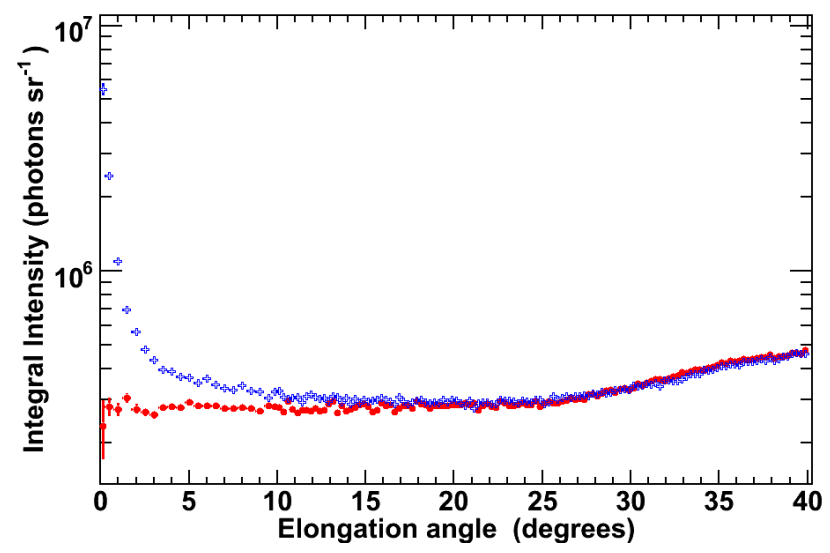

Figure 2. Integral intensity ( $\geq 100 \mathrm{MeV})$ plot for the Sun-centered sample vs. elongation angle, bin size: $0.25^{\circ}$. The upper set of data (open symbols, blue) represents the Sun, the lower set of data (filled symbols, red) represents the "fake-Sun" background.

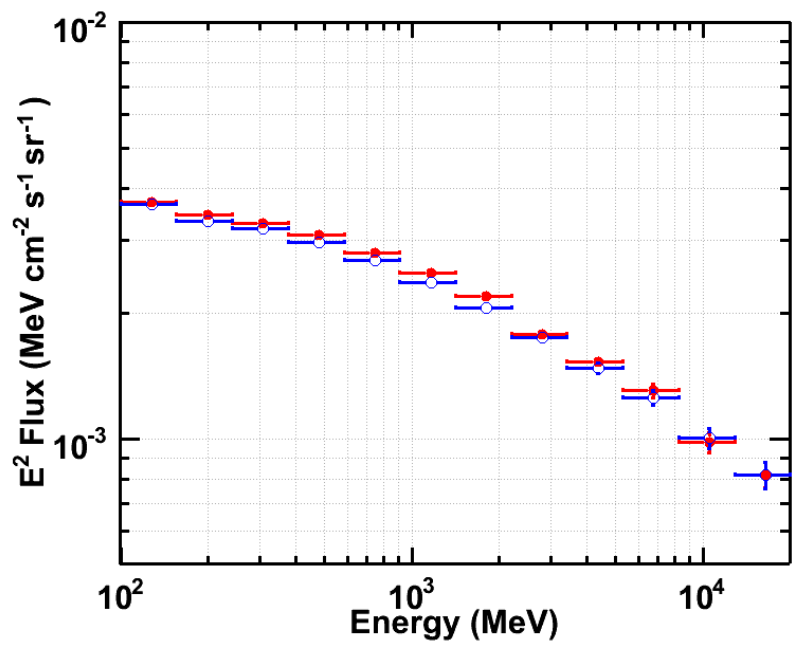

Figure 3. Reconstructed spectrum of the background for the fake-Sun method (filled symbols, red) and for the simulated background sample (open symbols, blue) averaged over a $20^{\circ}$ radius around the position of the Sun. 


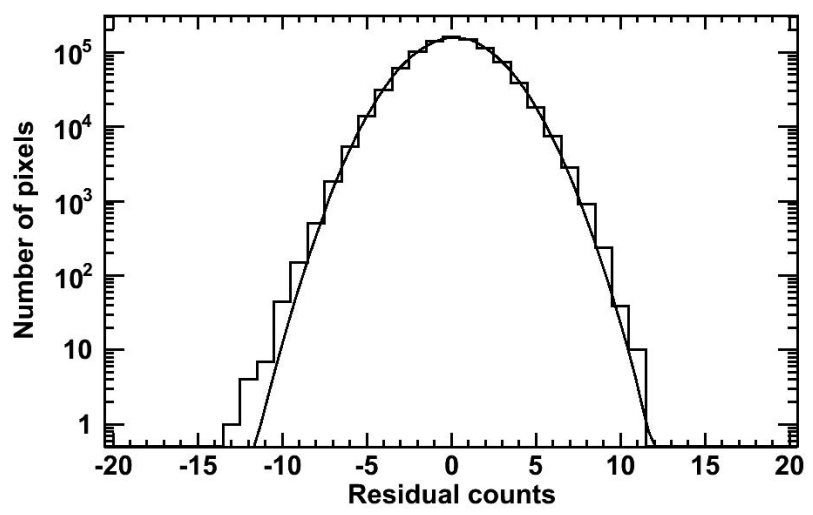

Figure 4. Distribution of the residual counts: differences between the photon counts in the map centered at the average fake-Sun and the high statistics count map derived from the model. A normal distribution with the same parameters is shown by the bold line. The map used to generate this distribution has a bin size of $0.3^{\circ}$ centered on the solar position of the simulated sample.
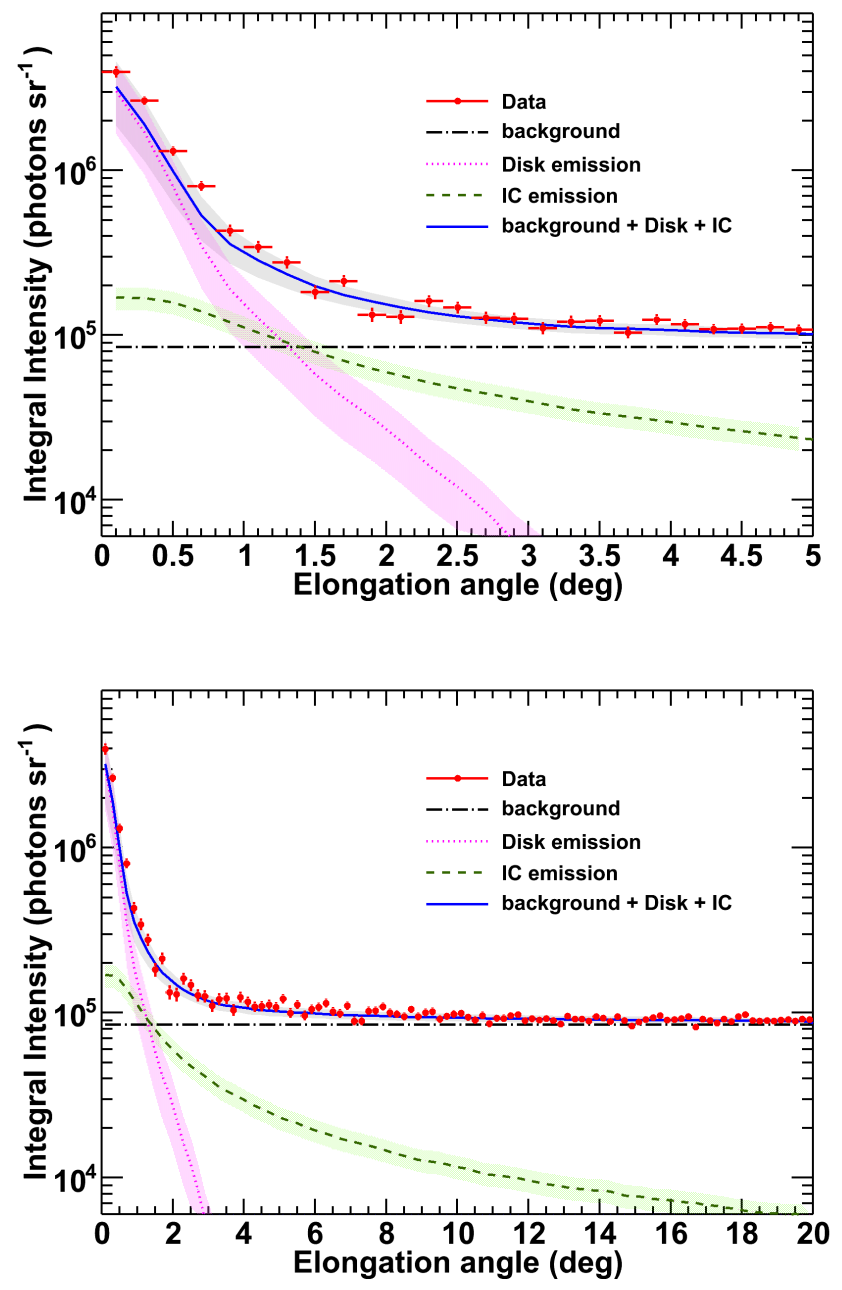

Figure 5. Integral intensity profiles above $500 \mathrm{MeV}$ for elongation angles $\leq 5^{\circ}$ (top) and $\leq 20^{\circ}$ (bottom). Points (red) are the observed counts, dash-dotted horizontal (black) line is the background, dotted (magenta) and dashed (green) lines are the point-like and extended components of the emission, correspondingly. The solid (blue) line is the sum of the background and the two components of the emission. The shaded areas around the lines show total error estimates. See text for details. 


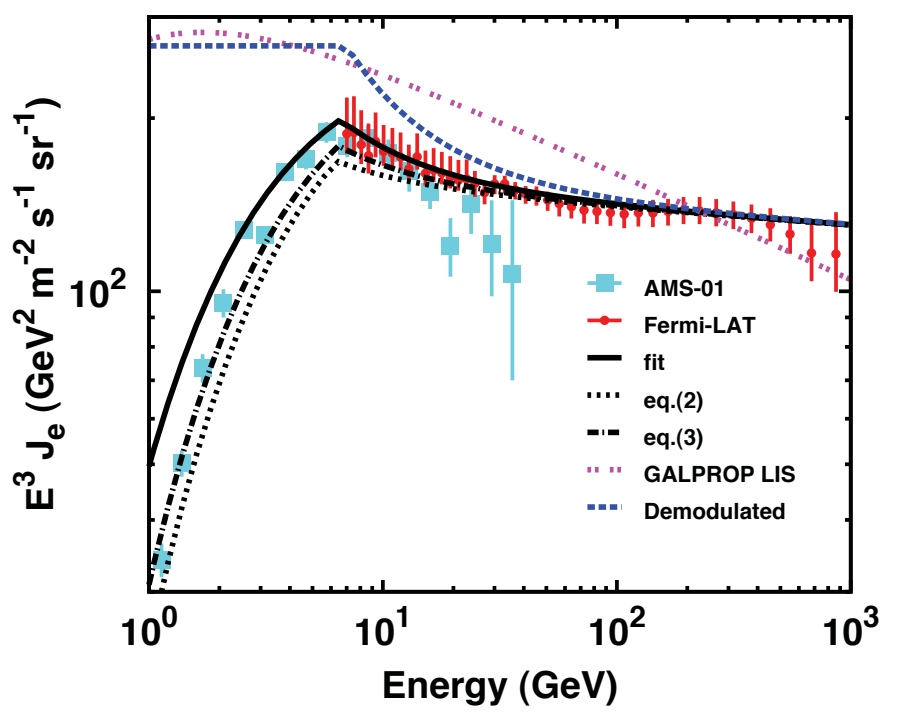

Figure 6. Electron spectrum used in the models of the IC emission compared with the data from Fermi-LAT (small red filled circles, Abdo et al. 2009b Ackermann et al. 2010) and AMS-01 (blue filled squares, Alcaraz et al. 2000). The thick solid (black) line is a fit to the Fermi-LAT electron spectrum at 1 AU; this is also the electron spectrum for $r<1 \mathrm{AU}$ in Model 3. The dash-dotted (black) and doted (black) lines show the electron spectra at at $r=0.3 \mathrm{AU}$ in Models 1 and 2 calculated for $\Phi_{0}=400 \mathrm{MV}$, eq. (2) and eq. (3), correspondingly. The dashed (blue) line shows the demodulated local interstellar spectrum. The double-dot (magenta) line shows the local interstellar spectrum of leptons as calculated by GALPROP (Ptuskin et al. 2006); this spectrum is used in the model calculations of the diffuse Galactic emission.

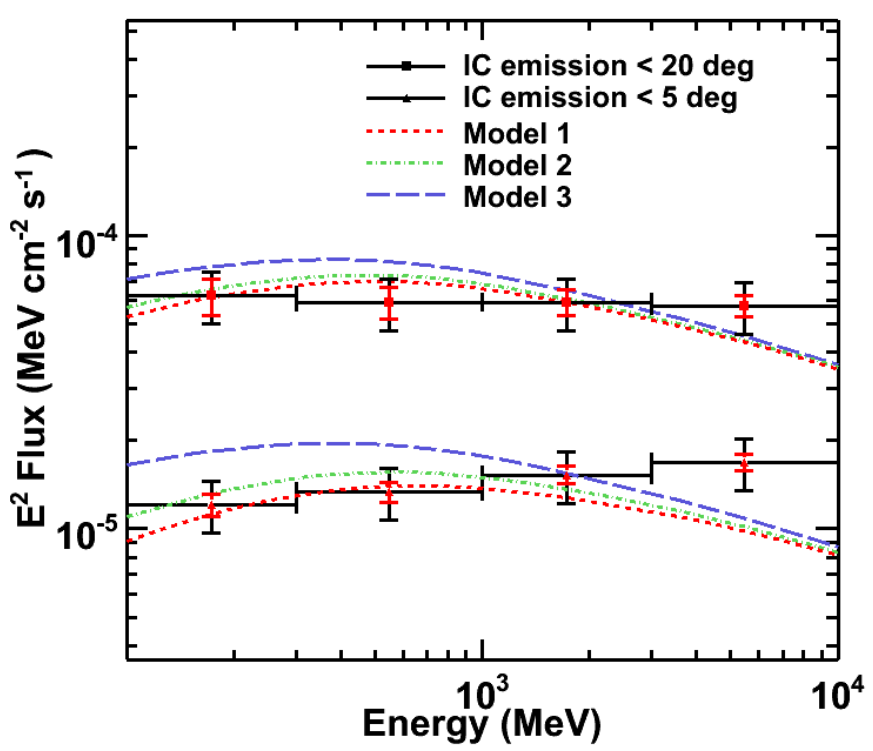

Figure 7. Energy spectra of the IC emission for elongation angles $\leq 5^{\circ}$ and $\leq 20^{\circ}$ as observed by Fermi-LAT and compared with model predictions. Statistical error bars (larger) are shown in black; systematic errors (smaller) are red. 


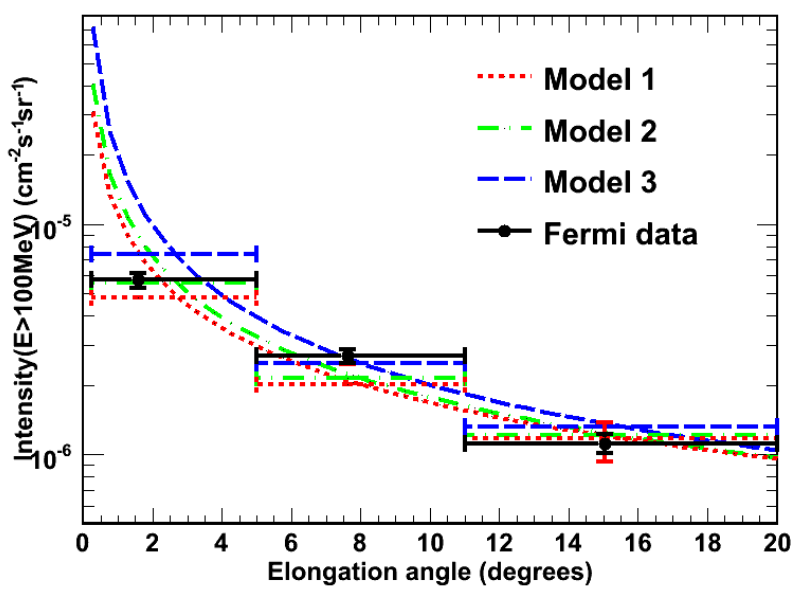

Figure 8. Intensity profile for the IC component vs. elongation angle compared with the model predictions. Statistical error bars (smaller) are shown in black; systematic errors (larger) are shown in red. To allow a direct comparison with the models, the model predictions are also shown binned with the same bin size as used for data.

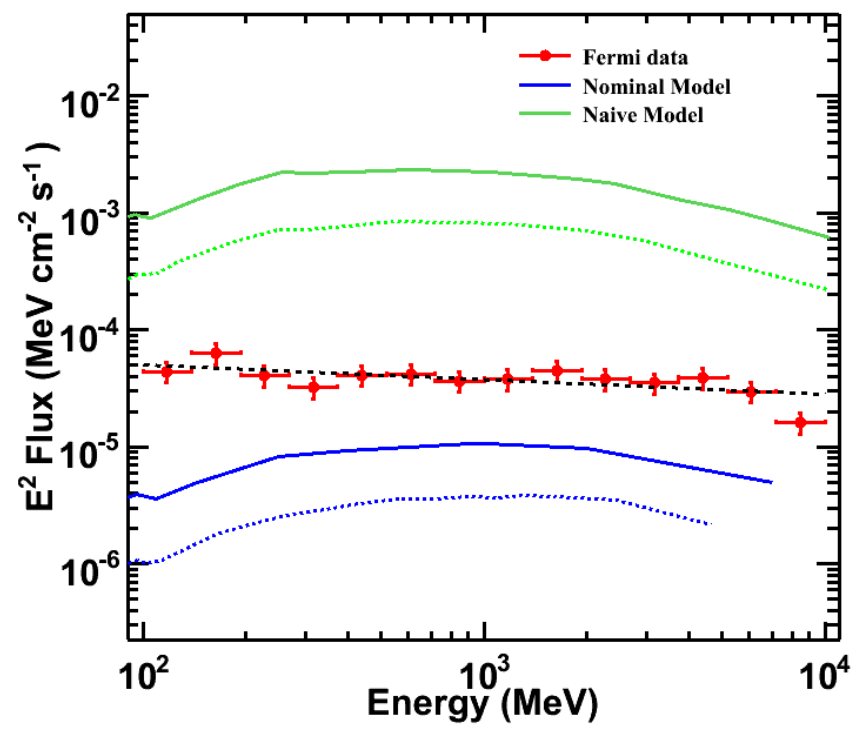

Figure 9. Energy spectrum for the disk emission as observed by the Fermi-LAT. The curves show the range for the "nominal" (lower set, blue) and "naive" (upper set, green) model predictions by Seckel et al. (1991) for different assumptions about CR cascade development in the solar atmosphere (see text for details). The black dashed line is the power-law fit to the data with index $2.11 \pm 0.73$. 